\title{
Pigmentation palatine due à la chloroquine persistant après plus de 50 ans?
}

\author{
Carla Patricia Martinelli-Kläy ${ }^{1}$, Tommaso Lombardi' ${ }^{1}$, Jacky Samson², * \\ ${ }^{1}$ Laboratoire d'Histopathologie buccale, Rue Barthélemy Menn 19, 1205 Genève, Suisse \\ ${ }^{2}$ Division de Stomatologie et Chirurgie orale, Rue Barthélemy Menn 19, 1205 Genève, Suisse \\ *jacky.samson@unige.ch
}

(Reçu le 15 mars 2012, accepté le 16 avril 2012)

Mots clés :

pigmentation muqueuse / chloroquine / palais

Key words:

mucosal pigmentation / chloroquine / palate
Résumé - Les antipaludéens de synthèse de référence - la chloroquine et l'hydroxychloroquine - ont vu leurs indications s'élargir après la découverte de leurs propriétés antiinflammatoires et immuno-modulatrices. Ils sont fréquemment utilisés pour le traitement de certaines affections dermatologiques et rhumatologiques. Ils sont le plus souvent bien tolérés mais ils comportent des effets indésirables, en particulier cutanéo-muqueux qui se traduisent principalement par une pigmentation gris bleuté ou noirâtre. Cette pigmentation peut intéresser la peau, le lit unguéal et la muqueuse buccale, le plus souvent la fibro-muqueuse palatine. Elle résulte d'une stimulation de la mélanogenèse et d'une accumulation de mélanine dans le derme ou le chorion, parfois associée à des dépôts d'hémosidérine. Normalement, la pigmentation régresse en quelques mois après l'arrêt du traitement.

Nous rapportons un cas où la pigmentation palatine semble persister plus de 50 ans après l'arrêt du traitement.

\begin{abstract}
Chloroquine-induced pigmentation of the hard palate persisting for more than $\mathbf{5 0}$ years ? Synthetic antimalarials - chloroquine and hydroxychloroquine - are now more largely prescribed to treat dermatological and rhumatological diseases because of their anti-inflammatory and immuno-modulatory activities. Usually well tolerated they may be responsible of multiple undesirable side effects affecting particularly skin and mucosae. The most frequent of these is a blue-grey or blackish pigmentation of the skin, the nail bed and the oral mucosa, more commonly the palatal fibromucosa. This pigmentation is considered to be the result of a melanogenic stimulation and accumulation of melanin in the dermis or chorion, sometimes associated with ferric iron. Usually the pigmentation regresses some months after the discontinuation of the antimalarial drug.

We report an unusual case of palatal pigmentation that seemed apparently to persist for more than 50 years after chloroquine discontinuation.
\end{abstract}

Un patient âgé de 66 ans est venu consulter en novembre 2007 pour une lésion médio-palatine pigmentée, remarquée dernièrement par une hygiéniste dentaire. Il s'agissait d'un patient en bonne santé, d'origine anglaise, installé depuis peu de temps dans la région genevoise, qui ne présentait pas d'antécédents médico-chirurgicaux. Il ignorait l'existence de cette plage pigmentée symétrique, aux limites nettes, d'environ $3 \mathrm{~cm}^{2}$, de couleur brunâtre irrégulière avec des zones discrètement violacées, qui semblaient parcourues par de petits vaisseaux, et quelques petites zones de muqueuse d'aspect normal; il n'y avait pas de limites nettes entre ces zones (Fig. 1). Devant cette lésion isolée et l'absence de renseignements précis, plusieurs diagnostics ont été envisagés : lésion d'origine vasculaire, mélanome, infiltration tumorale... L'examen histopathologique a montré qu'il n'y avait pas d'inflammation et que la fibro-muqueuse palatine était le siège d'une migration de pigment mélanique, confirmé par la coloration de Masson-Fontana ; le pigment était présent sous forme de grains de petite taille et de taille moyenne dans le chorion papillaire et réticulaire, et dans le cytoplasme des macrophages. Il n'y avait pas de pigment dans l'épithélium et l'assise basale semblait dépourvue d'activité mélanocytaire. La coloration de Perls a mis en évidence la présence de sels ferriques. Cette pigmentation mélanique du chorion de la muqueuse palatine, associée à des dépôts d'hémosidérine, faisait avant tout suspecter une pigmentation d'origine 


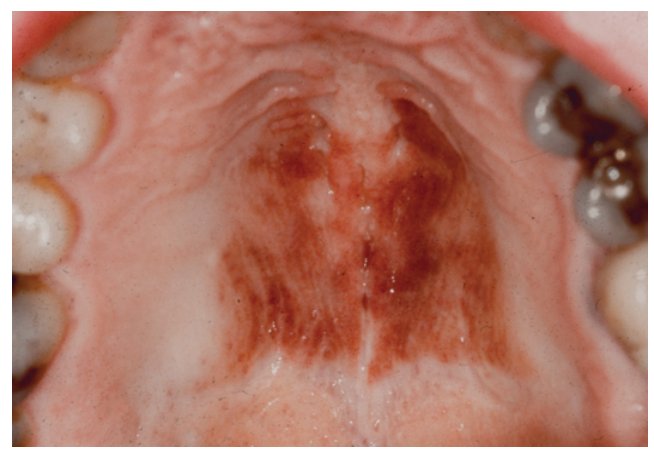

Fig. 1. Plage pigmentée médio-palatine, étendue, symétrique, bien limitée, de couleur brunâtre irrégulière, avec des zones discrètement violacées ou de couleur normale.

Fig. 1. Large pigmented palatal area, symmetrical, well limited, of irregular brownish color with slightly purplish zones or of normal color

médicamenteuse. En reprenant l'anamnèse et en informant le patient de l'hypothèse diagnostique, ce dernier s'est souvenu qu'ayant vécu en Afrique subsaharienne de l'âge de 6 à 12 ans, c'est-à-dire de 1947 à 1953, il a pris pendant ce séjour, à titre préventif, de la Nivaquine ${ }^{\circledR}$ et de la Paludrine ${ }^{\circledR}$.

\section{Commentaires}

Les antipaludéens de synthèse de référence - le sulfate de chloroquine ou Nivaquine ${ }^{\circledR}$ et le sulfate d'hydroxychloroquine ou Plaquenil ${ }^{\circledR}$-, initialement réservés au traitement préventif et curatif du paludisme, ont vu rapidement leur indication se diversifier avec la découverte de leurs propriétés antiinflammatoires et immuno-modulatrices. Ils sont prescrits dans le traitement de certaines affections dermatologiques (principalement le lupus érythémateux et les lucites mais aussi dans la porphyrie cutanée tardive, la sarcoïdose, la dermatomyosite ...) et rhumatologiques (principalement la polyarthrite rhumatoïde ...). Ils sont bien tolérés mais, surtout avec les traitements prolongés, on peut observer de multiples effets indésirables : digestifs - le plus fréquemment à type de nausées ou de diarrhées -, ophtalmologiques - le plus grave sous forme de rétinopathie -, dermatologiques, neuromusculaires, hématologiques, cardiovasculaires et toxicité embryonnaire et fœtale. Tout traitement prolongé avec les anti-paludéens de synthèse nécessite donc une surveillance biologique et ophtalmologique (bilan préthérapeutique et contrôles réguliers) [3].

Les effets indésirables cutanéo-muqueux, les plus fréquents après les effets indésirables digestifs, se traduisent surtout par une pigmentation cutanéo-muqueuse avec mélanonychies. On peut également observer des toxidermies prenant différents aspects : pustulose exanthématique aiguë généralisée, syndromes de Stevens-Johnson ou de Lyell, exacerbation d'un psoriasis, photosensibilité induite [3]. La pigmentation, allant du gris bleuté au noirâtre, se traduit par des macules de couleur assez homogène, à limites irrégulières, uniques ou multiples, plus ou moins étendues; elle peut intéresser la peau, le lit unguéal et la muqueuse buccale. Dans la cavité buccale, la pigmentation siège le plus souvent sur la fibro-muqueuse palatine $[1,2,4-7]$ - les premiers cas de pigmentation palatine dus aux antipaludéens (quinacrine) ont été rapportés en 1945 chez 156 soldats américains stationnés dans le Pacifique Sud [in 5, in 6] -, plus rarement la gencive, les joues, la langue ou les lèvres. La pigmentation est due à la présence de mélanine [7] ou d'hémosidérine dans le chorion, les deux étant le plus souvent associées $[3,5,6]$. Généralement, on considère que cette pigmentation régresse en grande partie ou disparaît totalement en quelques mois après l'arrêt du traitement. Certains auteurs [4] signalent toutefois une persistance prolongée, au moins 12 ans dans un cas [6]. Le mécanisme de cette pigmentation mélanique est inconnu (stimulation des mélanocytes, augmentation du taux des androgènes ? [3, 6]) mais il apparaît évident que les antipaludéens de synthèse ont une affinité particulière pour la mélanine ce qui peut expliquer leurs effets indésirables pour les cellules rétiniennes; devant l'apparition d'une pigmentation cutanéo-muqueuse, il est donc conseillé de réaliser un examen ophtalmologique $[3,5]$.

Le cas rapporté semble bien correspondre à une pigmentation palatine due à la chloroquine. L'examen histopathologique a orienté vers une pigmentation d'origine médicamenteuse et la chloroquine est le seul médicament consommé par ce patient susceptible d'avoir entrainé une pigmentation palatine. L'anamnèse et l'examen clinique ont permis d'éliminer toute autre étiologie. Cependant, ce cas est atypique par sa couleur et surtout sa persistance plus de 50 ans après l'arrêt du traitement mais aussi par l'absence de pigment et d'activité mélanocytaire dans l'épithélium en regard de la lésion.

\section{Conflits d'intérêt : aucun}

\section{Références}

1. Brasil Cda M, Ribeiro CM, Fonseca DD, Gueiros LA, Leao JC. Chloroquine-induced hyperpigmentation of the hard palate. Gen Dent 2012;60:e74-78.

2. De Melo Filho MR, Da Silva CA, Da Rocha Dourado M, De Oliveira Pires MB. Palate hyperpigmentation caused by prolonged use of the anti-malarial chloroquine. Head Neck Pathol 2012;6:48-50.

3. Fardet $L$, Revuz J. Antipaludéens de synthèse. Ann Dermatol Venereol 2005;132:665-74.

4. Gallo CB, Luiz AC, Ferrazzo KL, Migliari DA, Sugaya NN. Drug-induced pigmentation of hard palate and skin due to chronic chloroquine therapy: report of two cases. Clin Exp Derrmatol 2009;34:e266-7.

5. Kleinegger $\mathrm{CL}$, Hammond $\mathrm{HL}$, Finkelstein MW. Oral mucosal hyperpigmentation secondary to antimalarial drug therapy. Oral Surg Oral Med Oral Pathol Oral Radiol Endod 2000;90:189-94.

6. Lerman MA, Karimbux N, Guze KA, Woo SB. Pigmentation of the hard palate. Oral Surg Oral Med Oral Pathol Oral Radiol Endod 2009;107:8-12.

7. Moraes PC, Noce CW, Thomaz LA, Cintra ML, Correa ME. Pigmented lichenoid drug eruption secondary to chloroquine therapy: an unusual presentation in lower lip. Minerva Stomatol 2011;60:327-32. 